\title{
纳米结构的过渡金属氮化物复合物储能材料
}

\author{
岳艳花 ${ }^{\dagger}$, 韩鹏献 ${ }^{\dagger}$ 董杉木, 张克军, 张传健, 商超群, 崔光磊 ${ }^{*}$ \\ 中国科学院青岛生物能源与过程研究所, 青岛 266101 \\ $\dagger$ 同等贡献 \\ * 联系人, E-mail: cuigl@ qibebt.ac.cn
}

2012-02-07 收稿, 2012-04-01 接受

国家重点基础研究发展计划(2011CB935703)、中国科学院百人计划、山东省自然科学杰出青年基金(JQ200906)和国家自然科学基金(20971077) 资助

\begin{abstract}
摘要日益凸显的能源安全与气候变化问题引发了人们对可再生能源的不解追求, 从而带 来储能电池的革命性发展。高性能储能电池应该具有高能量密度、高功率密度、高安全性能、 长使用寿命等诸多特征, 这就要求人们研究开发新型电极材料. 近年来, 纳米材料以其独特 的表面效应、小尺寸效应以及量子尺寸效应从而产生强大电荷储存能力引起人们的广泛关 注. 本文综述了近年来本课题组在过渡金属氮化物纳米复合材料用于储能领域的研究进展, 基于电子和离子混合传输理念和有利的电荷跃迁界面, 阐述了其在高性能锂离子电池和超 级电容器等方面的应用.
\end{abstract}

关键词

纳米复合物 过渡金属氮化物

锂离子电池 超级电容器
能源安全与气候变化已然成为制约人类文明可 持续发展的两大关键性因素。在我国，化石能源资源 人均资源拥有量仅为世界人均的 $56 \%$, 能源的紧缺 与国家经济建设快速发展之间的矛盾日益尖锐，加 之一次能源消耗给生态环境带来的严重威胁, 开发 可再生、无污染的新型能源成为我国以及世界各国应 对挑战、实现经济可持续发展的必然举措. 在造成能 源安全与环境污染的成因中, 人类的交通运输行为 首当其冲. 据统计, 它消耗了世界上一半的石油, 并 造成将近 $1 / 4$ 的温室气体排放, 发达国家表现更甚 ${ }^{[1]}$. 因此, 研究和开发新型低碳、环保交通工具成为世界 各国重点支持方向. 在众多新型交通工具中, 以动力 电池为“心脏”的电动汽车备受人们关注, 这样一来 就对动力电池的能量密度、功率密度和转换效率提出 了更高的要求, 并且成本控制也尤为重要. 因此有必 要研究其储锂机理, 从而开发出高性能锂离子电池 电极材料.

此外, 作为低成本高功率储能器件的一种, 超级 电容器由于充放电迅速、使用寿命长 (可达上万次)、
高功率密度而在诸多领域尤其在混合动力汽车、风电 和光伏电间隙能源的电量均衡等方面具有不可替代 的作用及发展潜力. 一般来讲, 它以具有高比表面积 的材料为活性物质通过物理吸附电荷和法拉第反应 的形式进行能量存储. 目前, 碳材料与过渡金属氧化 物是应用最为广泛的电容器电极活性材料.

纳米结构的金属氮化物复合物是近年来锂离子 电池负极材料研究的热点之一，具有嵌锂电位低、反 应高度可逆和容量较高等特点. 其中, 含锂过渡金属 氮化物 $\mathrm{Li}_{x} \mathrm{M}_{y} \mathrm{~N}(\mathrm{M}=\mathrm{Fe}, \mathrm{Co}, \mathrm{Ni}, \mathrm{Cu}, \mathrm{Mn})$ 是基于 $\mathrm{Li}_{3} \mathrm{~N}$ 快 离子导体材料而衍生发展起来, 具有良好的离子电 导性和价态可变性, 其中以 $\mathrm{Li}_{3-x} \mathrm{Cu}_{x} \mathrm{~N}$ 性能最为优越 (可逆容量 $>650 \mathrm{mAh} / \mathrm{g}$ ), 其次为 $\mathrm{Li}_{3-x} \mathrm{Co}_{x} \mathrm{~N}$ (可逆容量 $>560 \mathrm{mAh} / \mathrm{g}$ ), 但锂离子反复的嵌人/脱出会造成电极 材料结构坉塌, 从而影响电池的功率密度和循环性 能. 国内关于过渡金属氮化物的相关研究也取得诸 多进展, 复旦大学付正文课题组对 $\mathrm{Fe}_{3} \mathrm{~N}^{[2]}, \mathrm{Ni}_{3} \mathrm{~N}^{[3]}$, $\mathrm{CrN}^{[4]}$ 和 $\mathrm{VN}^{[5]}$ 等多种氮化物及其二元复合物 ${ }^{[6]}$ 做了 深人研究, 结果表明, 高价态的金属氮化物往往具有

英文版见: Yue Y H, Han P X, Dong S M, et al. Nanostructured transition metal nitride composites as energy storage material. Chin Sci Bull, 2012, 57, doi: 10.1007/s11434-012-5301-1 
较高的容量, $\mathrm{CrN}, \mathrm{VN}$ 的首次容量可以达到 1500 $\mathrm{mAh} / \mathrm{g}$, 远高于 $\mathrm{Fe}_{3} \mathrm{~N}$ 和 $\mathrm{Co}_{3} \mathrm{~N}(400 \mathrm{mAh} / \mathrm{g})$, 且掺杂后 金属氮化物 $\left(\mathrm{Cr}_{x} \mathrm{Fe}_{1-x} \mathrm{~N}\right)$ 的性能优于未掺杂氮化物电 极性能. 然而, 在实际使用中, 氮化铬等材料由于极 化现象严重, 导致应用性降低, 其原因主要是电极材 料的电子和离子的电导率低下 ${ }^{[7]}$.

提高电池的能量密度关键是获得具有高能量密 度的电极材料, 而提高材料的比能量可通过如下方 法: 一是提高材料的容量, 二是降低材料的脱嵌理电 位 ${ }^{[8]}$. 前面已经提到, $\mathrm{VN}$ 在诸多过渡金属氮化物中 容量性质优异, 可以达到 $1500 \mathrm{mAh} / \mathrm{g}$, 远高于氮化 铁、氮化钴等, 因而对于研究高能量密度的储能材料 具有非常重要的意义.

提高电池的倍率性能关键是获得具有优异导电 性能的电极材料. 众所周知, 氮化钛是一种优良的导 电材料, 关于其在锂离子电池中的应用研究也有诸 多报道，如 $\mathrm{Li}_{4} \mathrm{Ti}_{5} \mathrm{O}_{12} / \mathrm{TiN}^{[9]}, \mathrm{Si} / \mathrm{TiN}^{[10]}$ 等材料经氮化 钛复合后倍率性能均得以改善. 最近, 有报道对氮化 钛/石墨烯的电催化性质进行了研究, 通过在石墨烯 表面形成 $\mathrm{C}_{3} \mathrm{~N}_{4}$, 之后引人钛源的方法制备得到氮化 钛/石墨烯. 研究结果表明, 该复合材料在染料敏化 太阳能电池以及生物传感器方面发挥出优异的性 能 ${ }^{[11]}$.

基于以上研究成果与存在的问题, 本课题组从
材料设计的理念出发就 VN, TiN 两种过渡金属氮化 物及氮化物复合物在锂离子电池以及超级电容器中 的应用进行了研究, 本综述将对相应结果进行介绍.

\section{1 过渡金属氮化物复合物用作锂离子电池 负极材料}

一般来讲, 锂离子电池充放电时存在 3 个关键性 动力学过程: (a) 离子在电解液本体中的扩散; (b) 离 子在电解液/界面的转移; (c) 离子在电极材料本体中 扩散; 如图 1 所示. 其中, (c) 是决定性步骤, 即材料 的离子和电子两者的传输难易程度决定了锂离子电 池性能的发挥. 受离子扩散方程的约束, 锂离子在材 料中的扩散时间 $(\tau)$ 与扩散长度 $(L)$ 的平方成正比, 即, $\tau=L^{2} / 2 D$ ( $D$ 为锂离子在固态材料中的扩散系数). 当 电极材料尺寸变小时, 锂离子在电极材料中的扩散 时间减少, 从而动力学性能大大改善 ${ }^{[12 \sim 14]}$

纳米电极材料具有比表面积大、锂离子嵌人脱出 深度小和行程短的优点, 在高倍率充放电时极化小、可 逆性良好、循环次数长, 并且锂离子反复嵌入/脱出时, 不易产生结构均塌 ${ }^{[15 ~ 17]}$. 作为一种性能优异的锂二 次电池电极材料, 既要能够实现快速的锂离子传输, 又要具有快速的电子传导特性 ${ }^{[18,19]}$. 因此, 我们设计 了一系列纳米结构混合传输(电子和离子)电极材料

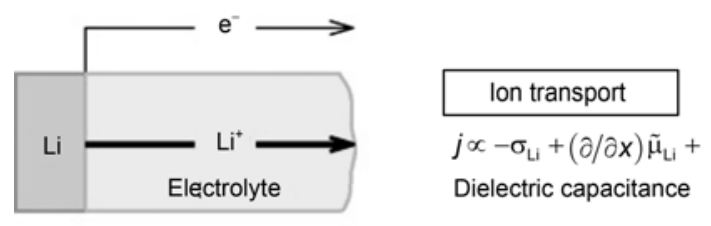

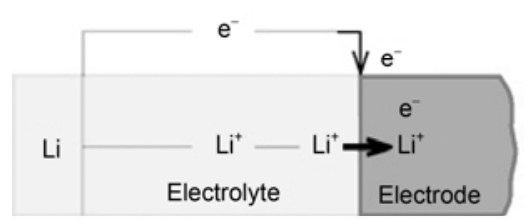
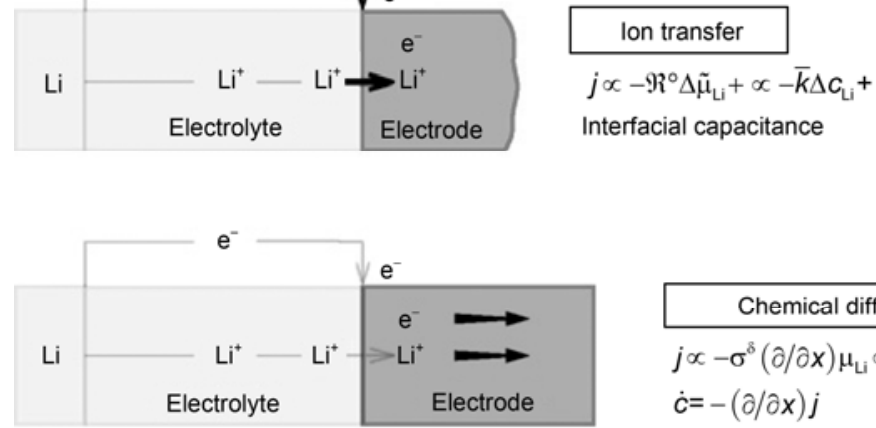

图 1 锂离子在锂离子二次电池中 3 个主要的动力学过程 Reprinted from [12], Copyright 2007, with permission from Elsevier 
(如图 2 所示), 同时构筑有利的界面传输网络, 研究 锂离子和电子的快速输运行为, 从而实现高能量密 度、高功率密度的锂离子电池电极材料.

\section{1 纳米结构 Ti-V-N/C 复合物用于锂离子电池 负极材料}

基于图 2 纳米结构的混合传输电极材料设计理 念, 本课题组就过渡金属氮化物复合材料做了较为 系统的研究. 已有文献对 $\mathrm{GeN}^{[20]}$ (氮化锗)、 $\mathrm{Zn}_{3} \mathrm{~N}_{2}{ }^{[21]}$ (氮 化锌)、 $\mathrm{Cu}_{3} \mathrm{~N}^{[22]}$ (氮化铜)等不同过渡金属氮化物 $\mathrm{M}_{x} \mathrm{~N}_{y}(\mathrm{M}$ 为过渡金属)的储锂行为进行了深人研究, 并提出储锂机理为:

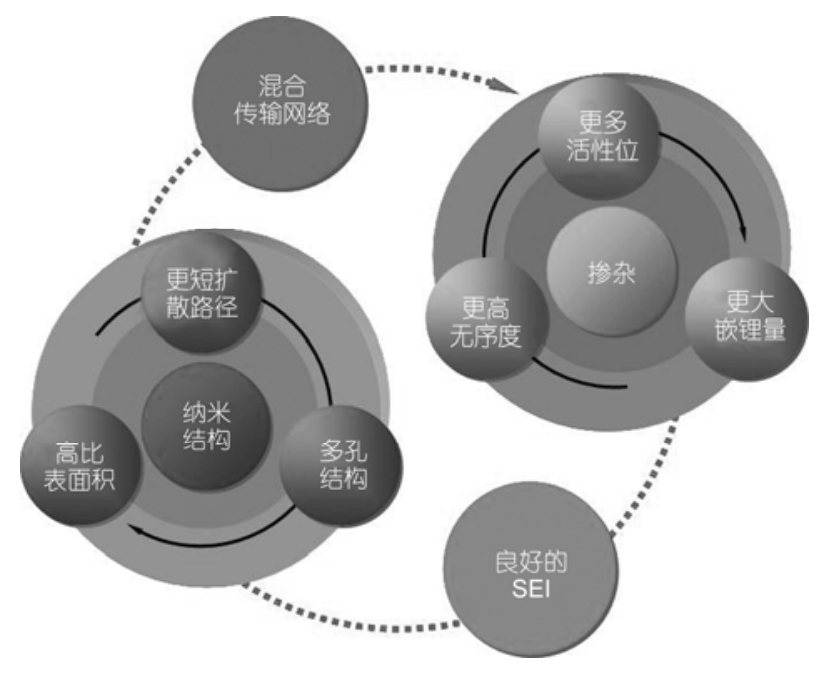

图 2 基于混合传输的纳米结构电极材料设计理念

$$
\begin{gathered}
\mathrm{M}_{x} \mathrm{~N}_{y}+3 y \mathrm{Li}^{+}+3 y \mathrm{e}^{-} \rightarrow x \mathrm{M}+y \mathrm{Li}_{3} \mathrm{~N} \\
\mathrm{M}+z \mathrm{Li}^{+}+z \mathrm{e}^{-} \rightarrow \mathrm{Li}_{z} \mathrm{M}
\end{gathered}
$$

一些氮化物室温时的电子电导率低下, 并且在 锂离子多次嵌入/脱出过程中体积变化大, 从而使得 电池功率密度和循环性能低下。一般来讲, 氮化钛 (TiN)具有良好的导电性和高温化学稳定性 ${ }^{[23]}$, 而文 献报道的氮化钒(VN)显示出巨大的储锂容量. 基于 此, 我们课题组和德国马普协会固态所 Joachim Maier 等教授合作，采用模板法合成了 Ti-V-N/C 纳米 复合材料 ${ }^{[24]}$ (如图 3 所示), 将两种氮化物的优势协同 发挥出来, 另外将碳包覆于表面, 可以有效吸收循环 过程由于锂离子嵌人/脱出带来的体积膨胀作用. 复 合材料的粒径在 $8 \mathrm{~nm}$ 左右, 储锂性能良好; 在电流 密度为 $74.4 \mathrm{~mA} / \mathrm{g}$ 时, 比容量达到 $639 \mathrm{mAh} / \mathrm{g}$; 即使 在 $22.32 \mathrm{~A} / \mathrm{g}$, 电极材料的比容量仍保持在 $95 \mathrm{mAh} / \mathrm{g}$, 表现出非常好的大电流充放电能力.

\section{2 石墨烯/过渡金属氮化物复合物用于锂离子 电池负极材料}

石墨烯一经出现即成为世界各国科研工作者的 研究热点. 石墨烯是一种由 $\mathrm{sp}^{2}$ 杂化碳原子构成的平 面纳米薄膜, 是一种单原子厚度的二维材料. 石墨烯 具有诸多物理化学优点, 如化学耐受性强、比表面积 大、电子导电性优异以及较宽的电化学窗口. 因此, 石墨烯在储能电池电极材料领域得到了广泛的研 究 ${ }^{25]}$. 我们课题组在氮掺杂对石墨烯结构与储锂物 性的构效关系(如图 4)以及相应的界面动力学行为方

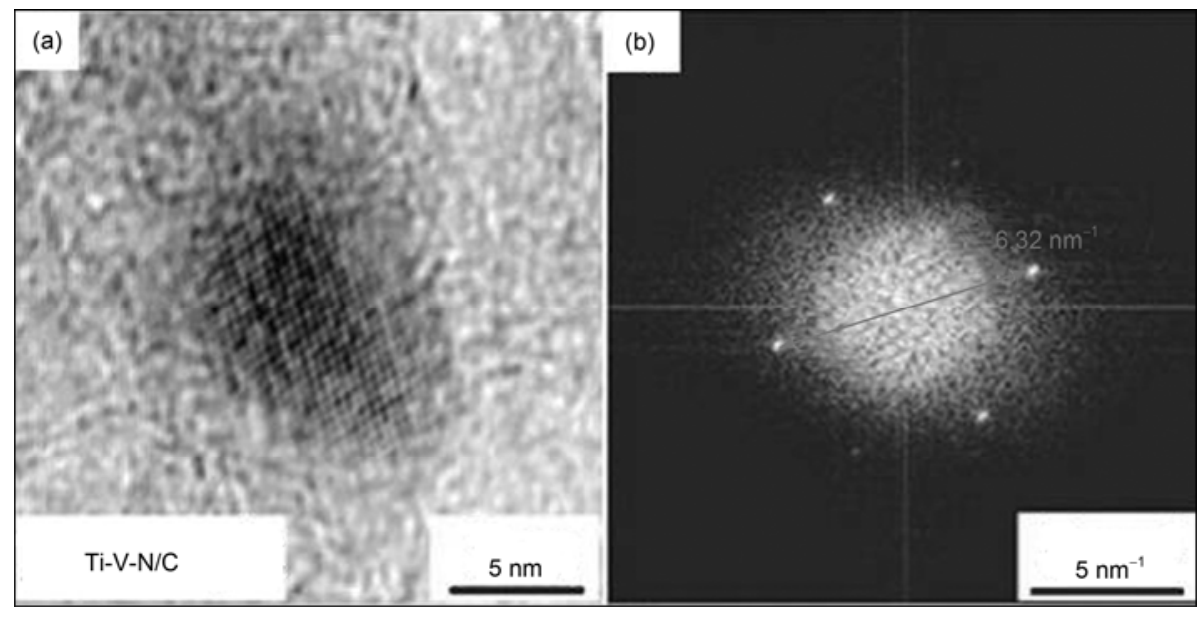

图 3 纳米结构的 Ti-V-N/C 复合材料

(a) 高倍率 TEM 图; (b) 电子衍射图. Reprinted with permission from [24] 


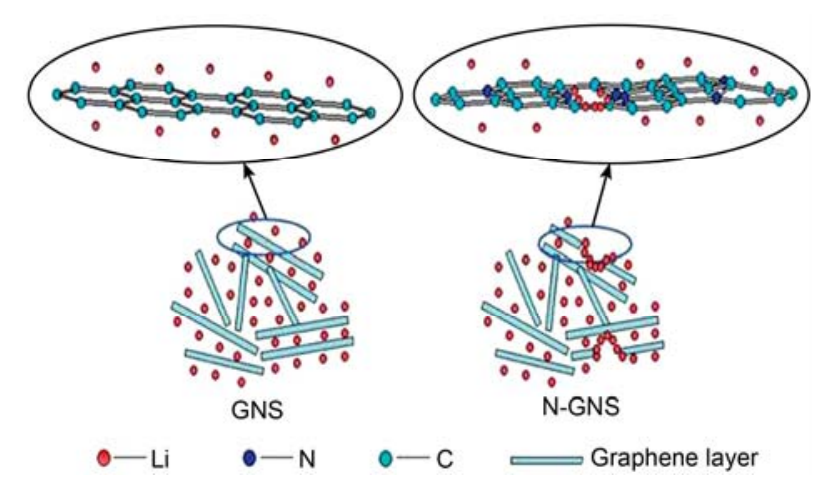

图 4 氮掺杂石墨烯储锂机制的示意图 Reprinted with permission from [26]

面做了相关研究工作. 研究表明, 氮掺杂为石墨烯片 层提供了更多锂活性位点, 尤其是吡啶型氮掺杂引 人的结构缺陷可以提高储锂容量, 同时氮的掺杂减 少了石墨烯边缘的催化位点从而降低不可逆锂的损 失, 改善材料的循环性能 ${ }^{[26]}$. 日本京都大学的 Ogumi 教授 ${ }^{[27]}$ 报道, 石墨类材料的界面阻抗很大, 因此减 少界面阻抗对于提高石墨类材料的动力学非常重要. 我们的研究结果表明, 氮的掺杂可以大大减少界面 阻抗, 提高动力学性能.

我们课题组在以上工作的基础上设计了石墨烯/ 氮化钛 $(\mathrm{G} / \mathrm{TiN})$ 复合物, 希望借由氮化钛纳米颗粒改 善石墨烯的表面性质, 二者共同构建良好的混合离 子传输网络, 以此提高石墨烯首次库仑效率, 增强材 料的倍率性能.

研究结果如图 $5^{[28]}$ 所示, 其中图 (a)为该材料所 制电极的首次充放电曲线, 单纯石墨烯的首次可逆 充电容量与首次库仑效率分别为 $438 \mathrm{mAh} / \mathrm{g}$ 和 $52 \%$,
而 $\mathrm{G} / \mathrm{TiN}$ 复合材料可提高至 $646 \mathrm{mAh} / \mathrm{g}$ 和 75\%, 这可 能是由于 $\mathrm{TiN}$ 纳米颗粒掩盖了石墨烯表面与不可逆 容量相关的部分缺陷位点, 从而提高了首次库仑效 率. 图(b)为电极的倍率性能曲线, 石墨烯经 $\mathrm{TiN}$ 复合 后倍率性能有了明显改善, 当电流密度达到 2000 $\mathrm{mA} / \mathrm{g}$ 时容量仍保持为 $325 \mathrm{~mA} / \mathrm{g}$, 远高于石墨烯在相 同倍率下的容量 $98 \mathrm{~mA} / \mathrm{g}$. 为了进一步探求 $\mathrm{G} / \mathrm{TiN}$ 复 合材料优良性能表现的原因, 我们利用电化学交流 阻抗以及等效模拟电路对材料的锂离子传输机理进 行了初步研究, 得出经复合后材料的 SEI 膜阻抗以及 电荷转移阻抗均有所降低, 这进一步论证了 $\mathrm{TiN}$ 与 石墨烯的复合有利于电池倍率性能的提高, 形成良 好的电极-电解液传输界面 ${ }^{[29,30]}$. 同时, 利用四探针 法测得 $\mathrm{G} / \mathrm{TiN}$ 的电导率为 $2.36 \mathrm{~S} / \mathrm{cm}$, 而石墨烯的电 导率为 $0.25 \mathrm{~S} / \mathrm{cm}$.

另外, 我们课题组对氮掺杂石墨烯/VN 复合物 用于锂离子电池负极材料做了较为深人的研究 ${ }^{[31]}$. 在研究氮掺杂石墨烯/VN 复合物的过程中, 发现复 合物的容量随着循环次数增多, 容量不断上升(如图 6(a)). 为了阐明容量上升的原因, 我们利用外场的 $\mathrm{XRD}$ (图 6(b))和高分辨透射电子显微镜技术(图 7)进 一步表征材料的充放电过程中性质的改变. 由 XRD 衍射图可以得到, 经充放电循环后, $\mathrm{VN}$ 在 $62^{\circ}$ 附近的 衍射特征峰发生向大角度移动的现象, 表明晶格尺 寸变小, 这可能是由于锂离子嵌人形成 $\mathrm{Li}_{x} \mathrm{~V}_{1-x} \mathrm{~N}$ 取 代原子半径较大的 $\mathrm{V}$ 引起的.

图 7(a)为氮掺杂石墨烯/VN 的高分辨透射电子显 微镜图, 可以看到 $\mathrm{VN}$ 晶粒尺寸在 20 100 nm 不等, 1, 2 区域为 $V N$ 晶粒，(b)即为对这两个区域所作的能
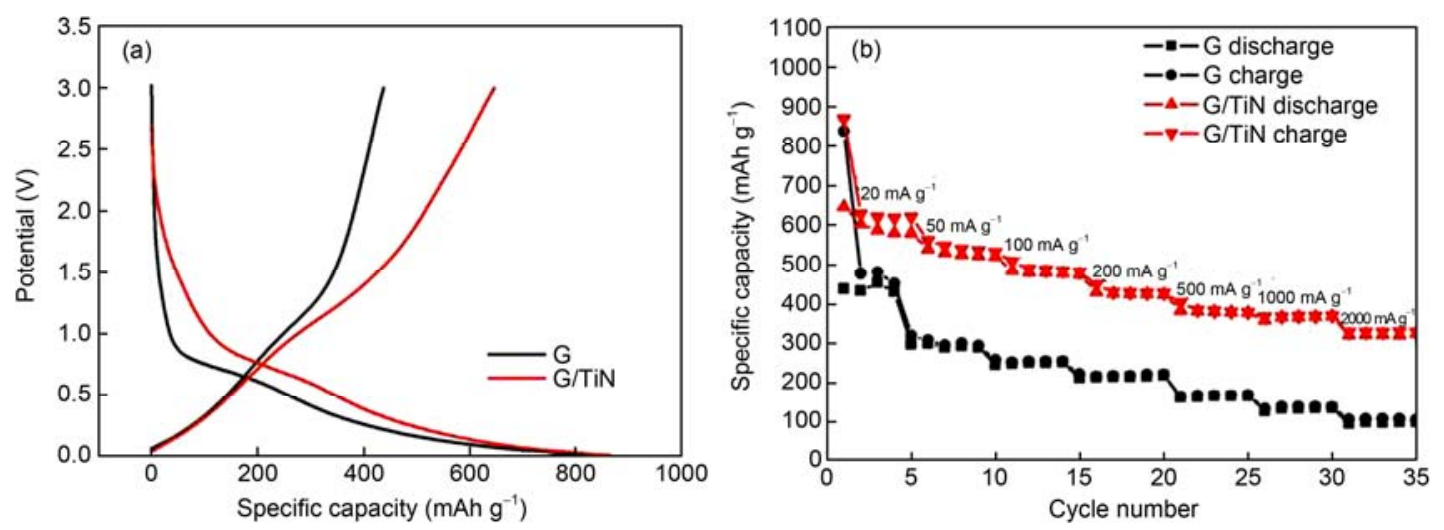

图 $5 \mathrm{G} / \mathrm{TiN}$ 的首次充放电曲线(a)与倍率性能曲线 $(\mathrm{b})$ Reprinted with permission from [28] 

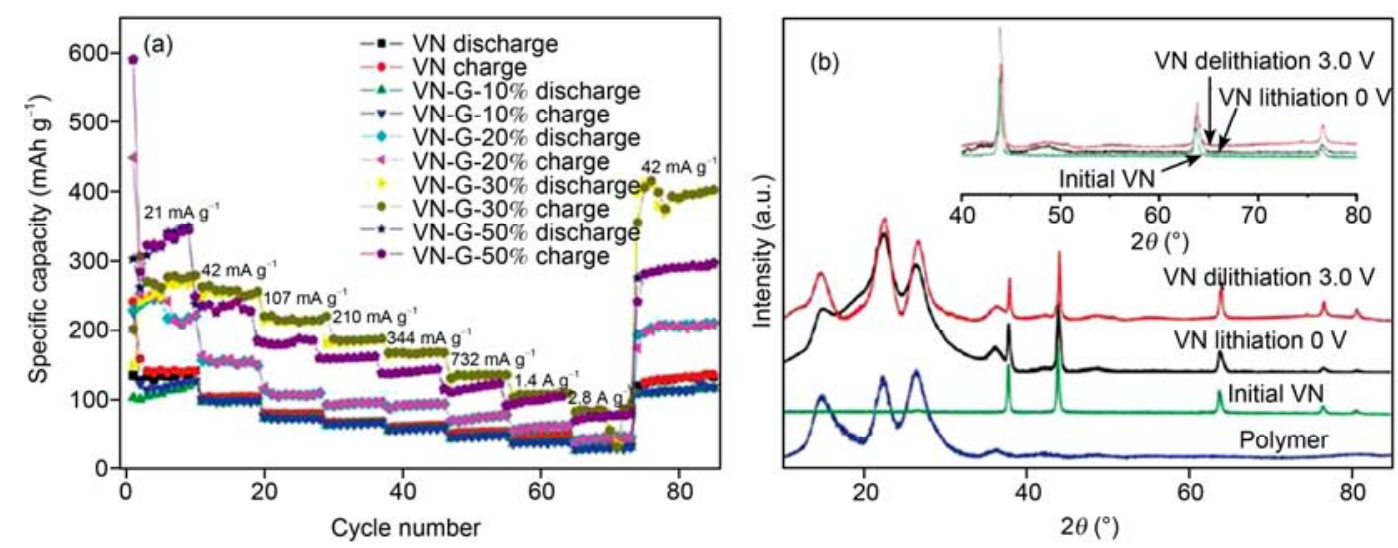

图 6 氮掺杂石墨烯/VN 复合物

(a) 倍率性能曲线图; (b) 不同电位下的 XRD 图. Reprinted with permission from [31]
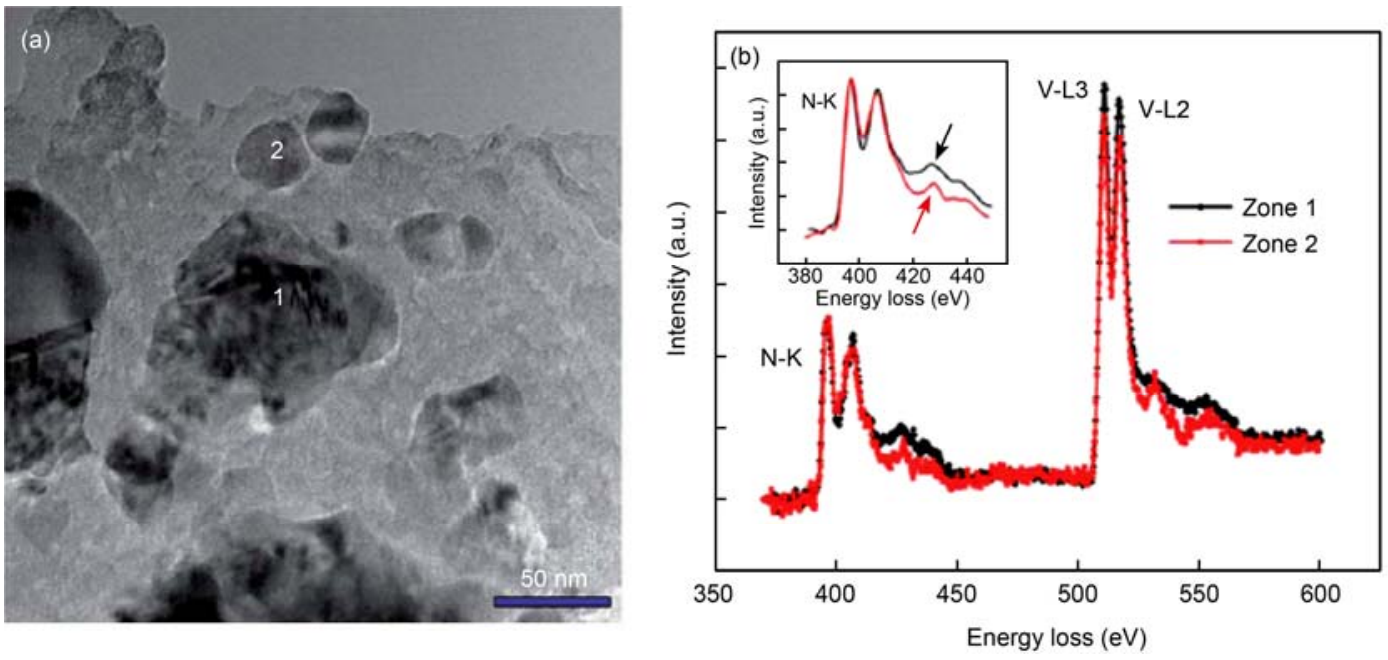

图 7 氮掺杂石墨烯/VN 复合物的 TEM 图(a)和 EELS 图(b)

Reprinted with permission from [31]

量损失谱. 通过对比发现, 1 区 $\mathrm{V}$ 元素的百分含量高 于 2 区，这表明锂离子嵌人 2 区的更多一些，尤其对 于较大尺寸 $\mathrm{VN}$ 晶粒锂离子的嵌人并不是短时间内 完成的, 而是随着循环次数的提高, 锂离子不断嵌人, 也就是说复合物被不断活化，从而导致可逆容量不 断提高.

锂离子电池电极材料结构纳米化后，很多不具 备储锂活性的材料表现出了比传统石墨材料更优越 的性能, 然而动力学性能的不足依然是制约因素. 为 了弥补这一不足, 我们通过设计可以实现离子与电 子有效传输的纳米结构材料, 建立新型界面储存电 荷等模型, 构建稳定的电极/电解液界面, 达到复合 电极材料的结构均一和可控制备，进而实现高比能
量、高功率、长循环寿命，因此，基于“混合传输”机 制的电极材料是将来动力锂离子电池的研发热点.

\section{2 过渡金属氮化物用于超级电容器}

研究具有高比表面积同时具备优良电子导电性 的材料是超级电容器研究领域的关键. 在过去几十 年里, 人们对介孔材料的制备做了大量研究. 介孔材 料通常具有较高的比表面积, 有效的可接触孔隙, 且 由于孔隙路径存在有利于离子迁移与传质的进行. 具有良好电子导电性的介孔氮化钛有望成为良好的 超级电容器活性材料. 同时, 非水相电容器由于较高 的工作电压, 通常具有较高的能量密度 ${ }^{[32]}$. 基于此, 我们课题组制备了具有介孔结构的 $\mathrm{TiN}$ 微球以及具 
有不同结构的 TiN-VN 纳米复合物, 并对其在超级电 容器方面的应用做了研究.

\section{1 介孔 TiN 微球用于超级电容器}

TiN 微球通常是将氧化钛进行氮化处理得到的, 但这种方法不易得到介孔结构, 模板法是获得介孔 氮化钛的常用方法. 然而, 模板法存在很大的缺陷, 制备过程耗时且在后续阶段需除去模板. 本课题组 提出一种非模板法介孔 $\mathrm{TiN}$ 微球的制备方法, 改变 合成条件借以改变微球产品尺寸(图 8). 纳米尺寸的 小球构成了微米尺寸的大球，纳米小球之间形成 20 $\mathrm{nm}$ 左右的孔道结构和强大的导电网络, 所形成的介 孔结构不仅增大了材料的比表面积, 更有利于电解 液在体系中的浸润与扩散. 电化学测试结果表明, 质 量比容量可高达 $112.3 \mathrm{~F} / \mathrm{g}(0.1 \mathrm{~A} / \mathrm{g}$ 的充放电电流密 度为 $0.1 \mathrm{~A} / \mathrm{g}$ ); 在功率密度为 $150 \mathrm{~W} / \mathrm{kg}$ 时, 能量密度 达到 $45 \mathrm{Wh} / \mathrm{kg}^{[33]}$.
介孔氮化钛球是非常有潜力的超级电容器电极 材料. 此外, 由于特有的介孔结构, 它可以作为三维 的集流体, 还可以根据需要引人 $\mathrm{MnO}_{2}, \mathrm{RuO}_{2}, \mathrm{Si}$ 和 $\mathrm{Sn}$ 等电活性物质, 从而构建三维混合传输网络, 这 类复合材料有望成为具有高比能量、高功率密度的电 极材料, 从而为高性能锂离子电池以及超级电容器 的研发应用注人新的活力.

\section{2 核壳结构的 TiN-VN 纳米线复合物用于超级 电容器}

前面已经提到，将氮化钒的高比容量性能与氮 化钛的优异导电性统一起来的可行办法即制备二者 的复合物, 从而建立高效的混合传输网络. 我们课题 组对二者的复合形式尤其是二者特定的纳米组装结 构进行了创新研究. 利用静电纺丝法, 采用套管式喷 头合成出皮芯结构 TiN-VN 纳米线介孔复合材料, 其 扫描电子显微镜结果如图 9 所示 ${ }^{[34]}$. 内层为 $\mathrm{VN}$, 外

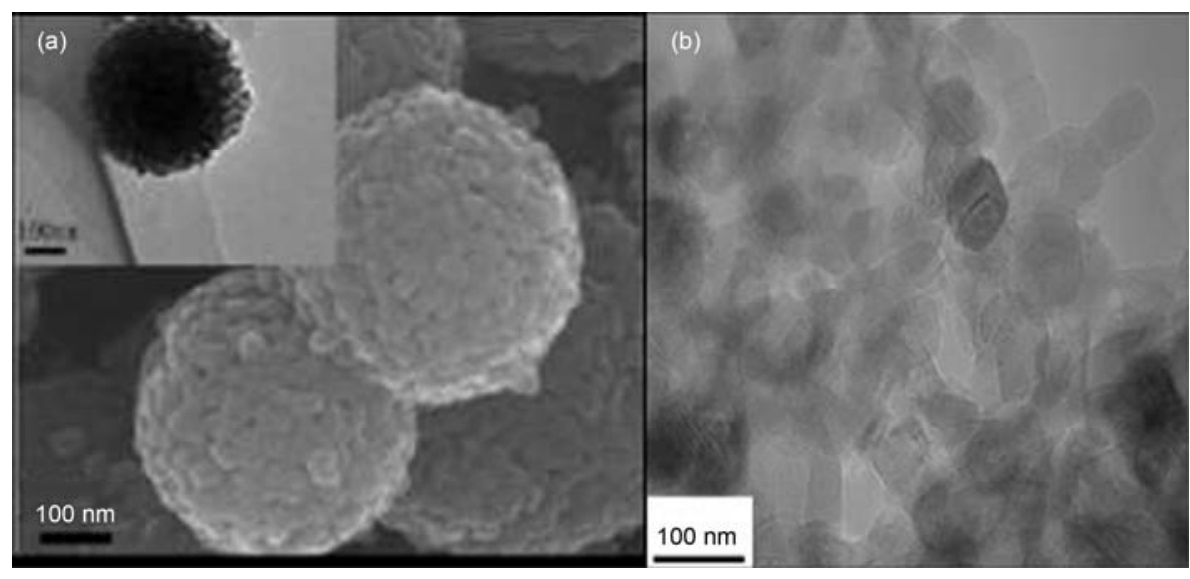

图 8 介孔 TiN 微球

(a) SEM 图; (b) 高分辨 TEM 图. Reprinted with permission from [33]

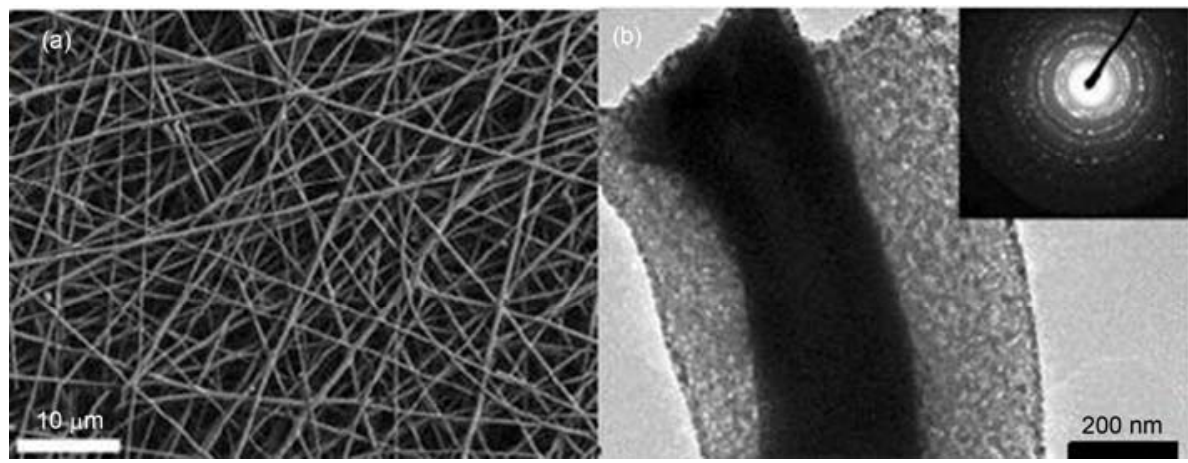

图 9 皮芯结构的 TiN-VN 介孔纳米线

(a) SEM 图; (b) TEM 图. Reprinted with permission from [34] 
层为介孔结构的 $\mathrm{TiN}$, 电化学测试结果表明, 在 2 $\mathrm{mV} / \mathrm{s}$ 扫描速率下, 质量比容量高达 $247.5 \mathrm{~F} / \mathrm{g}$, 即使 在 $50 \mathrm{mV} / \mathrm{s}$ 下, 比容量仍保持 $160.8 \mathrm{~F} / \mathrm{g}$, 表现出优越 的倍率性能.

本课题组利用两步合成法，对 $\mathrm{TiN}$ 纳米颗粒外 表面包覆氧化钒, 随之在氨气中进行高温氮化, 合成 出介孔核壳结构的 TiN-VN 球形纳米复合材料, 其电化
学性能优异, 制备及性能测试结果如图 10 所示 ${ }^{[35]}$

\section{3 一维 $\mathrm{MnO}_{2} / \mathrm{TiN}$ 纳米管阵列用于超级电容器}

目前，碳材料与过渡金属氧化物是应用最为广 泛的电容器电极活性材料. 作为过渡金属氧化物家 族中的一员, $\mathrm{MnO}_{2}$ 具有高能量密度、廉价易得等优势, 且天然储量丰富，使它成为最具商业化应用潜力的
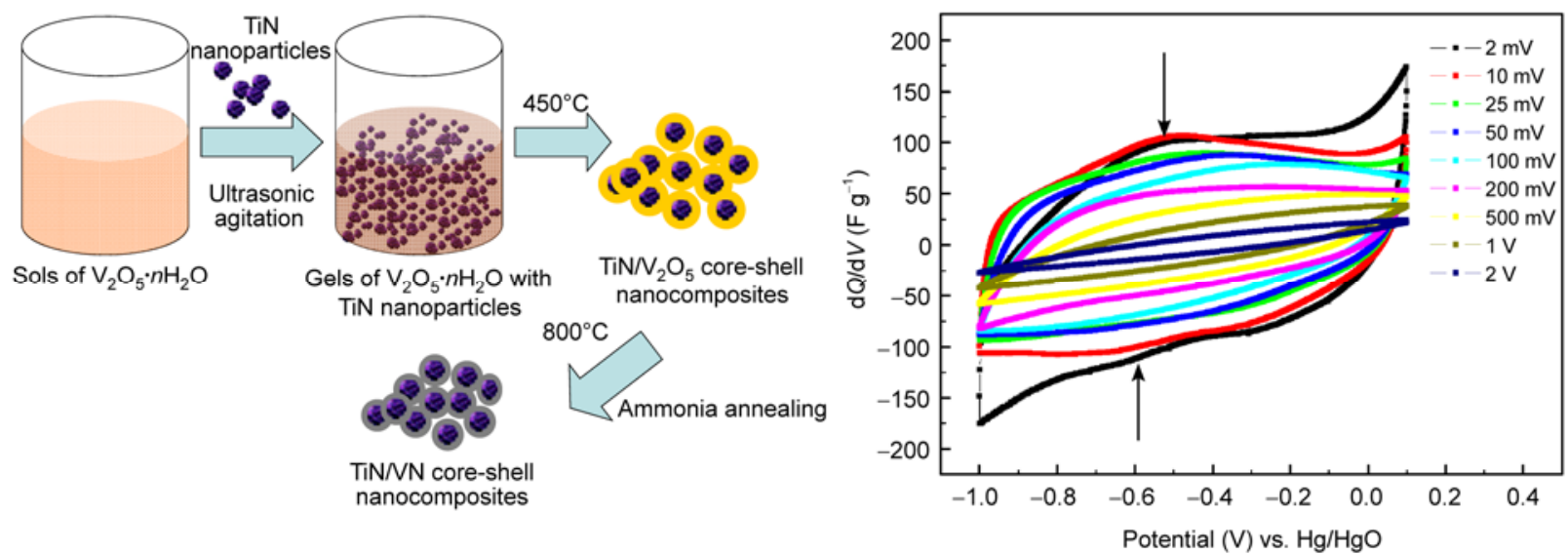

图 10 核壳结构的 TiN-VN 复合材料

(a) 制备示意图; (b) CV 曲线. Reprinted from [35]. Copyright 2011, with permission from Elsevier
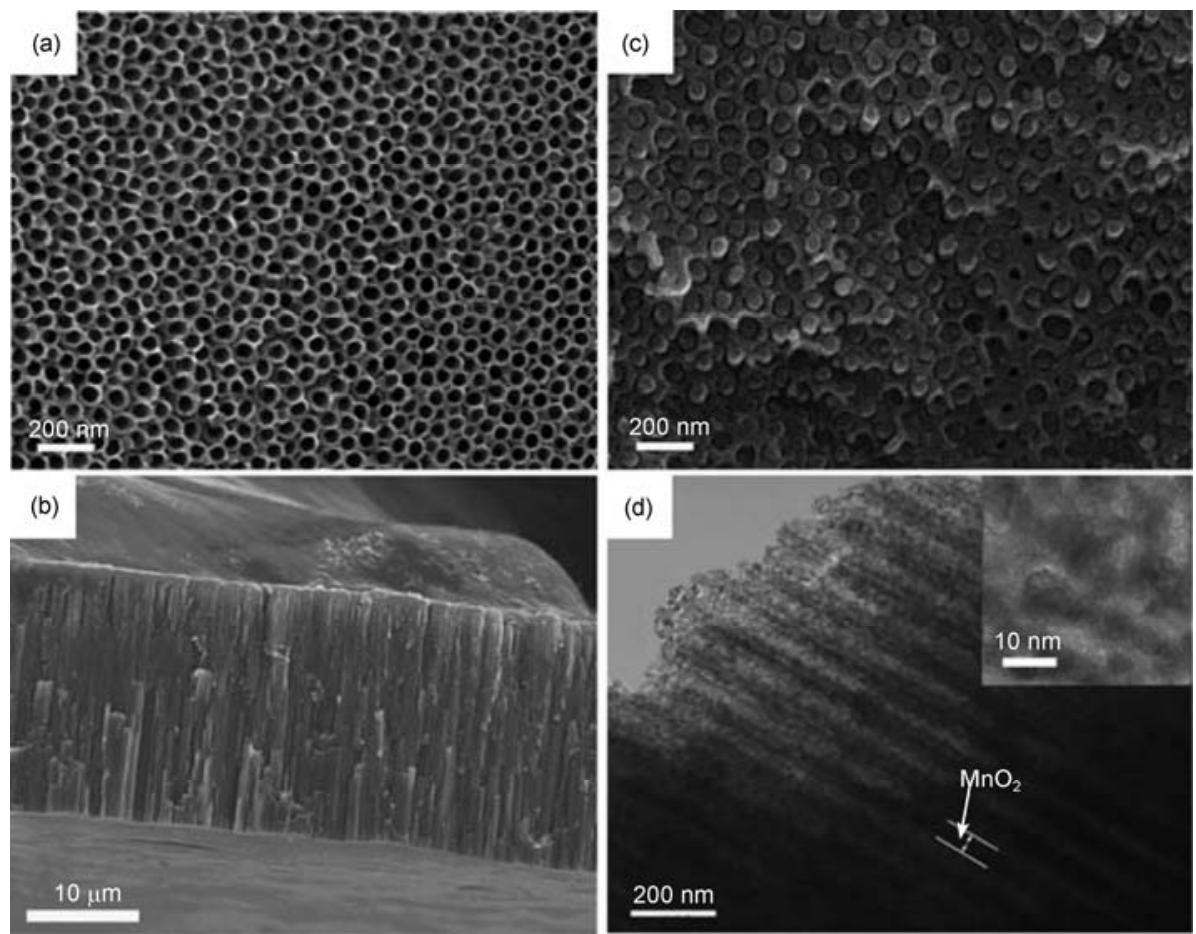

图 $11 \mathrm{TiN}$ 纳米阵列 $((\mathrm{a}),(\mathrm{b}))$ 和 $\mathrm{MnO}_{2} / \mathrm{TiN}$ 复合物 $((\mathrm{c}),(\mathrm{d}))$

Reprinted with permission from [36] 
候选材料之一. 然而, $\mathrm{MnO}_{2}$ 电子导电性差强人意, 在 大电流充放电过程中, 结构容易发生变化从而导致 电化学性能恶劣. 针对上述不足, 本课题组将 $\mathrm{MnO}_{2}$ 与高导电性 $\mathrm{TiN}$ 复合, 得到具有独特阵列结构的一 维 $\mathrm{MnO}_{2} / \mathrm{TiN}$ 纳米管复合材料. 图 11(a)和(b)分别为 制备的 TiN 纳米管阵列俯瞰与侧面的扫描电子显微 镜图, (c) 为在 $\mathrm{TiN}$ 纳米管阵列电沉积 $\mathrm{MnO}_{2}$ 后的扫描 电子显微镜图, (d)为该复合物的透射电子显微镜图, 可以看到, 电沉积的介孔 $\mathrm{MnO}_{2}$ 进人 $\mathrm{TiN}$ 纳米管的内 部. 复合材料的大电流充放电能力得到明显改善, 即 使在 $400 \mathrm{~A} / \mathrm{g}$ 的电流密度, 比容量仍高达 $390.2 \mathrm{~F} / \mathrm{g}$. 与 $2 \mathrm{mV} / \mathrm{s}$ 的扫描速率相比, $2000 \mathrm{mV} / \mathrm{s}$ 的容量保持率 达 $55 \%{ }^{[36]}$.

\section{3 结语与展望}

绿色高效储能器件的研究是当前新能源战略的 关键一环, 研究新型储能材料必然成为今后科研领 域的热点之一. 本课题组基于纳米结构的混合传输 材料的设计理念, 系统深人地研究了系列过渡金属 氮化物及其复合物的储能性能及其构效关系，通过 有利界面的构建以及特定纳米组装结构的实现，过 渡金属氮化物复合物表现出良好的容量性质以及倍 率性能，且该类材料可加工性强. 随着当前对兼具高 能量密度及高功率密度电源器件的需求不断攀升, 尤其对于锂离子电池与超级电容器, 过渡金属氮化 物复合物具有重要的研究意义与应用前景.

\section{参考文献}

1 严定中. 新型能源替代化石能源的有效途径一低碳燃料排放标准. 城市, 2010, 1: 74-75

2 Fu Z W, Wang Y, Yue X L, et al. Electrochemical reactions of lithium with transition metal nitride electrodes. J Phy Chem B, 2004, 108: 2236-2244

3 Wang Y, Fu Z W, Yue X L, et al. Electrochemical reactivity mechanism of $\mathrm{Ni}_{3} \mathrm{~N}$ with lithium. J Electrochem Soc, 2004, 151: E162

4 Sun Q, Fu Z W. An anode material of CrN for lithium-ion batteries. Solid-State Lett, 2007, 10: A189-A193

5 Sun Q, Fu Z W. Vanadium nitride as a novel thin film anode material for rechargeable lithium batteries. Electrochim Acta, 2008, 54: 403-409

6 Sun Q, Fu Z W. $\mathrm{Cr}_{1-x} \mathrm{Fe}_{x} \mathrm{~N}(0 \leqslant \mathrm{X} \leqslant 1)$ ternary transition metal nitrides as anode materials for lithium-ion batteries. Electrochem SolidState Lett, 2008, 11: A233-A237

7 孙乾. 磁控溅射制备高价氮化物并用于锂离子电池电极材料的研究. 硕士学位论文, 上海: 复旦大学, 2009

8 辛森, 郭玉国, 万立俊. 高能量密度锂二次电池电极材料研究进展. 中国科学: 化学, 2011, 41: 1229-1239

9 Snyder M Q, Trebukhova S A, Ravdel B, et al. Synthesis and characterization of atomic layer deposited titanium nitride thin films on lithium titanate spinel powder as a lithium ion battery anode. J Power Sources, 2007, 165: 379-385

10 Kim I, Kumta P N, Blomgren G E. Si/TiN nanocomposites: New anode materials for Li-ion batteries. Electrochem Solid-State Lett, 2000, 3: 493-496

11 Wen Z, Cui S, Pu H, et al. Metal nitride/graphene nanohybrids: General synthesis and multifunctional titanium nitride/graphene electrocatalyst. Adv Mater, 2011, 23: 5445-5450

12 Maier J. Size effects on mass transport and storage in lithium batteries. J Power Sources, 2007, 174: 569-574

13 Aurbach D, Bruneel J L, Grondn J, et al. Common electroanalytical behavior of Li intercalation processes into graphite and transition metal oxides. J Electrochem Soc, 1998, 145: 3024-3034

14 Balaya P. Size effects and nanostructured materials for energy applications. Energy Environ Sci, 2008, 1: 645-654

15 Linkov I, Steevens J. Nanomaterials: Risks and Benefits. Heidelberg: Springer, 2009

16 Li H, Wang Z X, Chen L Q, et al. Research on advanced materials for Li-ion batteries. Adv Mater, 2009, 21: 4593-4607

17 Guo Y G, Hu J S, Wan L J. Nanostructured materials for electrochemical energy conversion and storage devices. Adv Mater, 2008, 20: 2878-2887

18 Gaberscek M, Jamnik J. Impact of electrochemical wiring topology on the kinetics of insertion electrodes. J Solid State Ionics, 2006, 177: $2647-2651$

19 Guo Y G, Hu Y S, Maier J. Synthesis of hierarchically mesoporous anatase spheres and their application in lithium batteries. Chem Commun, 2006, 2783-2785

20 Pereira N, Balasubramanian M, Dupont L, et al. The electrochemistry of germanium nitride with lithium. J Electrochem Soc, 2003, 150: A1118-A1128 
21 Pereira N, Klein L C, Amatucci G G. The electrochemistry of $\mathrm{Zn}_{3} \mathrm{~N}_{2}$ and LiZnN. J Electrochem Soc, 2002, 149: A262-A271

22 Pereira N, Dupont L, Tarascon J M, et al. Electrochemistry of $\mathrm{Cu}_{3} \mathrm{~N}$ with lithium. J Electrochem Soc, 2003, 150: A1273-A1280

23 Harrison J F. Electronic structure of the transition metal nitrides TiN, VN, and CrN. J Phys Chem, 1996, 100: 3513-3519

24 Cui G L, Gu L, Thomas A. A carbon/titanium vanadium nitride composite for lithium storage. ChemPhysChem, 2010, 11: 3219-3223

25 Geim A K, Novoselov K S. The rise of graphene. Nat Mat, 2007, 6: 183-191

26 Wang H B, Zhang C J, Cui G L, et al. Nitrogen-doped graphene nanosheets with excellent lithium storage properties. J Mater Chem, 2011, 21: 5430-5434

27 Ogumi Z. A. C. impedance analysis of electrochemical lithium intercalation into highly oriented pyrolytic graphite. J Power Sources, 1997, 68: 227-231

28 Yue Y H, Han P X, Cui G L, et al. In situ synthesis of graphene/titanium nitride hybrid material with highly improved performance for lithium storage. J Mater Chem, 2012, 22: 4938-4943

29 庄全超, 陈作锋, 董全峰, 等. 石墨负极首次阴极极化过程的电化学阻抗谱研究. 科学通报, 2006, 51: 17-20

30 田雷雷, 庄全超, 李佳, 等. 锂离子在石墨烯材料中的嵌人脱出机制. 科学通报, 2011, 56: 1431-1439

31 Zhang K J, Wang H B, Cui G L, et al. A hybrid material of vanadium nitride and nitrogen-doped graphene for lithium storage. J Mater Chem, 2011, 21: 11916-11922

32 Jang B Z, Liu C G, David N, et al. Graphene surface-enabled lithium ion-exchanging cells: Next-generation high-power energy storage devices. Nano Lett, 2011, 11: 3785-3791

33 Dong S M, Chen X, Cui G L, et al. Facile preparation of mesoporous titanium nitride microsphres for electrochemical energy storage. ACS Appl Mater Interface, 2011, 3: 93-98

34 Zhou X H, Shang C Q, Cui G L, et al. Mesoporous coaxial titanium nitride-vanadium nitride fibers of core-shell structures for high-performance supercapacitors. ACS Appl Mater Interface, 2011, 3: 3058-3063 Dong S M, Chen X, Cui G L, et al. TiN/VN composites with core/shell structure for supercapacitors. Mater Res Bull, 2011, 46: 835-839 Dong S M, Chen X, Cui G L, et al. One dimensional $\mathrm{MnO}_{2}$ /titanium nitride nanotube coaxial arrays for high performance electrochemical capacitive energy storage. Energy Environ Sci, 2011, 4: 3502-3508 